\title{
Explorando Arquiteturas Pedagógicas Recomendadas por meio de Agentes e Ontologia de Modelo do Aluno em Ambientes Virtuais de Aprendizagem
}

\author{
Vitor Bremgartner ${ }^{1,2}$, José Francisco M. Netto ${ }^{1}$, Crediné Menezes ${ }^{3}$ \\ ${ }^{1}$ Instituto de Computação (IComp) - Universidade Federal do Amazonas (UFAM) \\ Av. Gen. Rodrigo Otávio, Coroado. Manaus - AM - Brasil \\ ${ }^{2}$ Instituto Federal do Amazonas (IFAM) - Campus Manaus Distrito Industrial \\ Av. Danilo Areosa, Distrito Industrial. Manaus - AM - Brasil \\ ${ }^{3}$ Faculdade de Educação - Universidade Federal do Rio Grande do Sul (UFRGS) \\ Av. Paulo Gama, Farroupilha. Porto Alegre - RS - Brasil \\ \{vitorbref, jnetto\}@icomp.ufam.edu.br, credine@gmail.com
}

\begin{abstract}
Typically, the existing resources in Virtual Learning Environments (VLEs), used in distance education courses and blended, are presented in the same way for all students. This may complicate the effective learning process of each student. The approach adopted for the solution of this problem in this paper is based on a framework called AMPARA, which allows adaptation of resources for students in VLEs and student monitoring during his course, allowing the construction of his knowledge, using multiagent system technology that handles an open learner model ontology. One of AMPARA resources is recommendation and adaptation of Pedagogical Architectures containing proposals for constructivist activities for the students have a more effective learning of a particular course content. Results obtained in a discipline of Algorithms and Programming show the feasibility of the proposal.
\end{abstract}

Resumo. Normalmente, os recursos existentes em Ambientes Virtuais de Aprendizagem (AVAs), utilizados em cursos de educação a distância e em semipresenciais, são apresentados da mesma forma para todos os alunos. Isto pode dificultar o processo de aprendizagem efetiva de cada aluno. A abordagem adotada para a solução deste problema neste artigo baseia-se em um arcabouço chamado AMPARA, que permite adaptação de recursos para alunos em AVAs e o acompanhamento do aluno no decorrer do seu curso, permitindo a sua construção do conhecimento, utilizando a tecnologia de sistema multiagente que manipula uma ontologia de modelo aberto de aluno. Um dos recursos do AMPARA é a recomendação e adaptação de Arquiteturas Pedagógicas contendo propostas de atividades construtivistas para os alunos terem uma aprendizagem mais efetiva do conteúdo de um determinado curso. Resultados obtidos em uma disciplina de Algoritmos e Programação mostram a viabilidade da proposta.

\section{Introdução}

A Educação a Distância (EaD) já é uma modalidade amplamente utilizada nos processos de ensinoaprendizagem. Para apoiar os cursos de EaD ou semipresenciais existem os Ambientes Virtuais de Aprendizagem (AVAs), facilitando o processo de comunicação entre estudantes, professores, tutores e a comunidade, permitindo a todos participarem de um modo interativo com a disponibilidade de materiais didáticos. Além disso, existem AVAs que utilizam uma camada de inteligência, especialmente no que diz respeito à possibilidade de ensino flexível para os alunos, em que o ambiente de aprendizagem é capaz de adaptar os seus recursos apresentados de modo a alcançar uma aprendizagem mais efetiva, de acordo com as necessidades do aluno [Bremgartner, Netto e Menezes 2014a]. Ferramentas na área da Inteligência Artificial, tais como ontologias e agentes de software podem atuar integrados nestes AVAs, sendo responsáveis por esta camada de inteligência e fazendo 
uso de um modelo do aluno [Viccari e Giraffa 2003]. A adaptação de recursos em AVAs pode ser entendida como a adaptação do que é apresentado para o aluno no AVA, como atividades passadas, objetos de aprendizagem, mudanças na interface e auxílio personalizado de acordo com o modelo de aluno, obtido através de seu perfil. Este modelo é um registro das ações dos alunos, bem como a informação útil a respeito do aluno no ambiente de aprendizagem. Como o perfil do aluno é constituído por dados brutos dos alunos no sistema, o modelo do aluno é composto de informações consideradas mais importantes do perfil do aluno que serão úteis para os processos de adaptação e personalização em AVAs. Por exemplo, estes dados podem ser informações pessoais, preferências e desempenho em atividades. Instituições e organizações internacionais têm estabelecido a padronização de modelo de aluno utilizado em AVAs, sendo que o padrão mais conhecido atualmente é o Instructional Management Systems Learning Information Package (IMS LIP) [LIP 2015].

No entanto, apesar da crescente utilização de ambientes educacionais, eles geralmente oferecem recursos de aprendizagem da mesma forma para todos os alunos, resultando que a aprendizagem pode não se tornar efetiva para todos, devido às diversas características cognitivas que cada aluno possui. Isto, portanto, cria dificuldades de aprendizagem para alguns alunos ou até mesmo a falta de interesse por parte dos alunos no uso do ambiente de aprendizagem. Além disso, apesar de existirem várias técnicas de adaptação de recursos de aprendizagem para alunos por meio de sistemas sensíveis ao contexto [Saccol et al. 2009], apoio a formação de grupos [Oliveira e Tedesco 2009] e personalização com agentes [Bastos Filho et al. 2006], muitos apresentaram importantes inovações tecnológicas, mas ainda existem desafios que necessitam de uma proposta pedagógica que possa promover o aprendizado efetivo do estudante em função de seu perfil.

Sendo assim, a abordagem adotada para solução deste problema neste artigo baseia-se em um arcabouço chamado AMPARA (do inglês, Adaptive Muti-agent Pedagogical Architectures for Resources and Activities in VLEs, que traduzindo fica Arquiteturas Pedagógicas Multiagente Adaptativas para Recursos e Atividades em AVAs), sendo uma estratégia que permite adaptação de recursos para alunos em AVAs e o acompanhamento do aluno no decorrer do seu curso, com base no Construtivismo de Piaget, utilizando a tecnologia de sistema multiagente que manipula uma ontologia de modelo aberto de aluno composto de várias características que o aluno possui, tais como competências, habilidades, equipamentos que o aluno utiliza, o desempenho nas atividades, frequência e estilos de aprendizagem. O padrão LIP foi estendido para integrar todas essas características do aluno. Os recursos de adaptação no AMPARA podem ser a recomendação de Arquiteturas Pedagógicas (APs) contendo propostas de atividades construtivistas com o objetivo de o aluno ter uma aprendizagem mais efetiva do conteúdo de um determinado curso. Além disso, o modelo de aluno é alterado dinamicamente durante o curso, através das interações do aluno com o AVA. O modelo de aluno também é apresentado ao estudante, sendo um Modelo Aberto de Aluno (MAA). Usar MAA refere-se a tornar o modelo de aluno explícito ao estudante, de modo a fornecer um recurso adicional através do autoconhecimento e possível autorregulação do processo de aprendizagem, com o objetivo de melhorar a aprendizagem e autonomia do aluno [Bull, Quigley e Mabbott 2006]. Utilizamos, portanto, como recurso de adaptação, a recomendação de APs que estimulam a colaboração entre estudantes, por meio de sistemas multiagente utilizando uma ontologia de MAA.

Além desta Introdução, o artigo está estruturado da seguinte forma: a Seção 2 discute a utlização de APs em AVAs. A Seção 3 apresenta trabalhos relacionados. A Seção 4 mostra os métodos empregados, uma visão geral do modelo conceitual do AMPARA, descrevendo também o sistema multiagente e o modelo de aluno. A Seção 5 apresenta testes realizados de adaptação de recursos e arquiteturas pedagógicas em uma disciplina de Algoritmos e Programação em um AVA. A Seção 6 apresenta as conclusões e trabalhos futuros. 


\section{Arquiteturas Pedagógicas em AVAs}

A compreensão acerca da expressão "Arquiteturas Pedagógicas" (APs) na área educacional tem trazido múltiplas interpretações, sendo que estas estão diretamente relacionadas com uma linha epistemológica que dá embasamento para sua proposta pedagógica [Behar, Bernardi e Silva 2009].

A AP pode ser compreendida como a construção de estratégias pedagógicas que tem como base uma determinada teoria e seus pressupostos a fim de auxiliar na efetivação da aprendizagem com suporte de recursos tecnológicos como os AVAs e/ou videoconferência. A construção da estratégia pedagógica envolve, entretanto, a formação de uma equipe interdisciplinar com a participação de profissionais das áreas de educação e computação.

Em outra perspectiva, partindo da concepção elaborada por [Carvalho, Nevado e Menezes 2005], APs podem ser compreendidas como "estruturas de aprendizagem realizadas a partir da confluência de diferentes componentes: abordagem pedagógica, software, Internet, inteligência artificial, educação a distância, concepção de tempo e espaço". Ou seja, são combinados os recursos tecnológicos com a visão pedagógica, sendo esta conjunção o elemento que fundamenta a AP. Para [Carvalho, Nevado e Menezes 2005], pressupõe-se que as APs são viabilizadas pela convergência entre os paradigmas epistemológicos e as estratégias pedagógicas, acolhendo, assim, uma possibilidade de releitura dessas, demonstrando-se, mais receptivas à aprendizagem. Assim, a construção das arquiteturas pedagógicas pode ser metaforicamente relacionada a uma atividade artesanal, onde será tecida uma rede de relações entre as experiências vivenciadas pelos sujeitos envolvidos e a reflexão sobre diferentes fatos e objetos relacionados com o meio de atuação em estudo. Este processo, entretanto, necessita de propostas pedagógicas abertas a uma abordagem didática flexível e adaptável aos mais diversos enfoques temáticos.

Ferramentas como wikis, blogs e fóruns de discussão, bem como a utilização de técnicas de pesquisa, permitem uma interatividade que supera as práticas convencionais, o que vem a agregar na construção de ambientes integradores, coletivos e colaborativos que podem ser utilizados como Arquiteturas Pedagógicas nos processos educativos [Marcon, Machado e Carvalho 2012]. Outros exemplos de APs são as atividades colaborativas debate de teses, controvérsia acadêmica e projetos de aprendizagem, apresentados em [Castro e Menezes 2011]. Neste trabalho, a AP utilizada foi o debate de teses, onde nessa arquitetura, a intenção é que o participante, partindo de seu conhecimento prévio, estenda e aprofunde seus conhecimentos através de interações com seus pares, seguindo uma determinada dinâmica. Nestas interações, que são realizadas através da produção de textos, os participantes expõem suas convicções sobre determinada(s) tese(s) proposta(s) pelo mediador.

\section{Trabalhos Relacionados}

O uso de Arquiteturas Pedagógicas em AVAs tem sido uma alternativa utilizada para apoiar os processos de ensino e aprendizagem mediados pela tecnologia. Em trabalhos recentes, pode-se ver a crescente utilização das APs. Em [Ferrari, Oliveira e Menezes 2014], é proposto um ambiente para suporte a projetos de aprendizagem (AproAp), um ambiente com ferramentas que contemplam a todas as etapas de desenvolvimento de um projeto de aprendizagem. Em [Marcon, Machado e Carvalho 2012], é explorada a capacidade de utilizar o Facebook para a elaboração de APs com atividades em grupo. Por sua vez, em [Panceri e Menezes 2014] é utilizada a AP do debate de teses e são identificadas mediações pedagógicas que podem ser praticadas em AVAs por meio de processamento de linguagem natural, recuperação de informação e um sistema multiagente denominado ALPES. Além disso, em [Corte Real e Corbellini 2011], é mostrado o uso de uma ferramenta Wiki (PBWORKS - pbworks.com) como AP, propiciando um ambiente cooperativo onde foram investigadas as relações que foram sendo constituídas e as suas implicações entre os participantes.

Ainda na literatura pesquisada, foram encontrados trabalhos que não utilizam explicitamente Arquiteturas Pedagógicas, mas que tratam de técnicas de adaptações de recursos e possibilidades construtivistas em AVAs. Como forma de superar o sentimento geral de isolamento e consequente alta evasão de alunos em AVAs, o trabalho de [Oliveira e Tedesco 2009] apresenta os 
resultados de um experimento com o modelo chamado $i$-Collaboration que promove a colaboração entre os usuários em AVA. O i-Collaboration baseia-se na utilização de agentes companheiros virtuais de aprendizagem. Por sua vez, o Sistema Inteligente de Desafios Abertos (IOCS) [Bastos Filho et al. 2006] consiste em um sistema multiagente que realiza a mediação entre o sistema e o usuário. O sistema tem a proposta de trabalhar a aprendizagem através da aplicação de desafios que consistem em problemas lógico-matemáticos de concepção aberta, preocupando-se em reconhecer como os alunos respondem as atividades propostas. Usando tecnologias móveis, o paradigma educacional considerado no projeto COMTEXT [Saccol et al. 2009] é interacionista-construtivista. As ferramentas dentro do ambiente de aprendizagem COMTEXT têm como objetivo apoiar o desenvolvimento de competências, promovendo a interação entre a comunidade de alunos, com foco no compartilhamento de conhecimento e o desenvolvimento de habilidades e atitudes.

Assim, podemos ver que existem vários trabalhos na literatura que lidam com Arquiteturas Pedagógicas, abordagens construtivistas, sistema multiagente e modelo de aluno aplicado em AVAs. No entanto, observou-se com estes trabalhos descritos acima que muitos destes estudos utilizam características específicas dos alunos de forma separada. Em outras palavras, alguns trabalhos lidam apenas com estilos de aprendizagem, outros com competências dos alunos; outros, apenas dependendo do equipamento que o aluno utiliza. Assim, o modelo de aluno não é capaz de informar ao AVA o perfil global do aluno e sua situação atual com maior precisão no ambiente de aprendizagem. Consequentemente, fica difícil para o AVA tomar decisões corretas na tentativa de auxiliar o aluno em suas necessidades reais. A proposta do AMPARA difere ao considerar várias características dos estudantes ao mesmo tempo, variando de acordo com a interação dos alunos com o AVA e também ao apresentar o modelo aberto de aluno para o mesmo, além de proporcionar adaptação e recomendação de APs e outros recursos no AVA para o estudante, em função de seu modelo de aluno. A próxima seção descreve os métodos empregados com mais detalhes.

\section{Métodos Empregados}

\subsection{Modelo Conceitual do AMPARA}

O modelo conceitual do AMPARA é mostrado na Figura 1. Neste modelo, é mostrado em detalhes o arcabouço que constitui o processo de personalização de atividades colaborativas baseadas em APs em função do modelo de aluno, fazendo uso do sistema multiagente. Os agentes foram desenvolvidos nos frameworks JADE [JADE 2015] e JADEX [JADEX 2015].

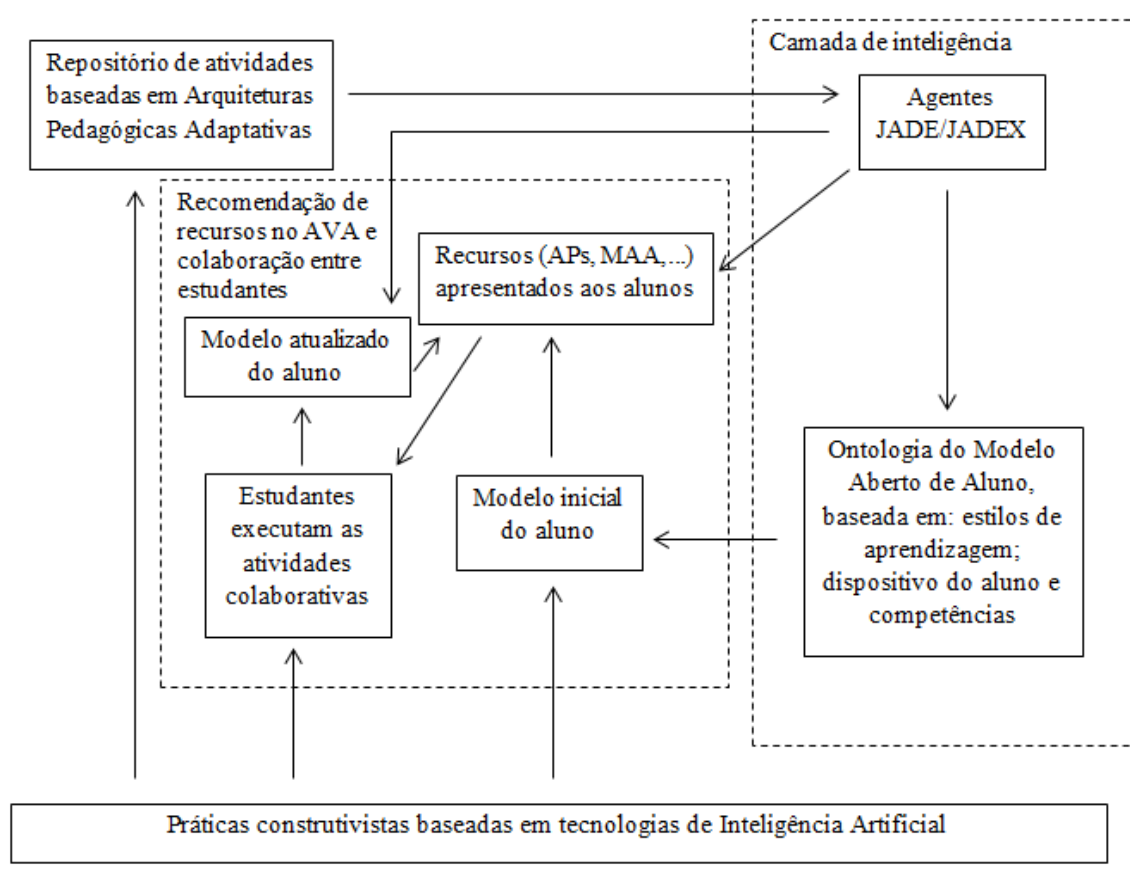

Figura 1. Modelo Conceitual do AMPARA. 
Nesse modelo conceitual, o processo de adaptação de recursos começa quando o professor prepara atividades, registra estudantes e acessa o AVA. O estudante, por sua vez, interage com o AVA, seja cadastrando os seus dados pessoais, postando algo no fórum ou resolvendo atividades propostas no ambiente. Para este trabalho, utilizamos o AVA Moodle [Moodle 2015]. Além disso, no AVA foram criados três questionários para os alunos responderem no início do curso, com o objetivo de obter o perfil e o modelo de aluno inicial, porque esta é a primeira informação que compõe a base de conhecimento sobre os alunos no banco de dados. Os questionários são: 1) Índice de Estilos de Aprendizagem (Index of Learning Styles), buscando conhecer os estilos de aprendizagem de Felder-Silvernam [Felder e Silverman 1988] dos estudantes; 2) Questionário de Estilos de Aprendizagem de Honey-Alonso [Alonso e Gallego 2015]. Optou-se por escolher estes dois questionários visando ter uma informação mais precisa a respeito dos alunos quanto aos seus estilos de aprendizagem, obtidos pela combinação dos dois questionários; 3) Questionário sobre algoritmos e programação de computadores, onde os alunos responderão a perguntas sobre o seu nível de conhecimento em conceitos de programação referentes a disciplina de Algoritmos e Programação (estruturas condicionais; repetição; estruturas de dados; nível de experiência no uso da linguagem de programação da disciplina - a linguagem C; experiência de trabalhar com linguagens de programação no ramo profissional). Os links para acessar estes questionários e para acessar o MAA ficam disponíveis no AVA. O AMPARA é uma extensão dos trabalhos de [Bremgatner, Netto e Menezes 2014a; Bremgartner, Netto e Menezes 2014b].

Por sua vez, a arquitetura geral do AMPARA é mostrada na Figura 2. Os agentes JADE manipulam os dados dos alunos e seus modelos de aluno no banco de dados do AVA, executando atualizações. O modelo de aluno é descrito por sua ontologia, que também é útil para a manipulação correta de troca de mensagens entre os agentes. Por sua vez, os agentes JADEX, a partir dos dados obtidos do modelo de aluno e usando o modelo Beliefs-Desires-Intentions (BDI) [Georgeff et al. 1999], selecionarão recursos construtivistas contidos no repositório (que fica no banco de dados), como por exemplo, APs, para adaptar o AVA. Finalmente, o aluno acessa o AVA adaptado em funçao de seu perfil e o seu MAA. Os agentes JADE, que são responsáveis por manipular os dados do modelo do aluno, são:

- Initial Profile Agent: configura os níveis de competências, habilidades e estilos de aprendizagem iniciais dos alunos em função das respostas dos alunos dos 3 questionários respondidos no início do curso e envia estas informações ao Update Profile Agent;

- Learning Assessment Agent: responsável pela avaliação das atividades respondidas pelos alunos, e envia as pontuações dos alunos ao Update Profile Agent;

- Update Profile Agent: responsável por atualizar os dados dos modelos de alunos mediante as interações dos mesmos com o ambiente, mediante as informações oriundas do Initial Profile Agent, Learning Assessment Agent e Frequency Evaluation Agent;

- Notifier Activity Agent: agente notificador de novas atividades passadas pelo professor, a fim de manter o aluno informado acerca de novas atividades;

- Frequency Evaluation Agent: agente que verifica a frequência do aluno no AVA. Convencionamos aqui que um aluno é muito frequente no AVA se ele faz mais de 3 acessos por dia, é normalmente frequente se acessa de 3 a 1 vez por dia, regularmente frequente se acessa pelo menos 1 vez por semana e não frequente caso contrário. Os dados de frequência do aluno serão enviados ao Update Profile Agent;

- Open Learner Model Agent: Apresenta ao professor quem são os alunos que discordam de seu modelo de aluno, a fim de que o professor possa tomar as medidas necessárias para atualização dos dados do aluno;

- Device Identifier Agent: responsável por detectar informações a respeito do equipamento que o estudante está utilizando, se é um computador, smartphone, tablet ou qualquer outro, a fim de ajustar o AVA de acordo com as características do dispositivo que o estudante estiver utilizando.

Os agentes JADEX, responsáveis pela recomendação de recursos aos alunos no AVA, são: 


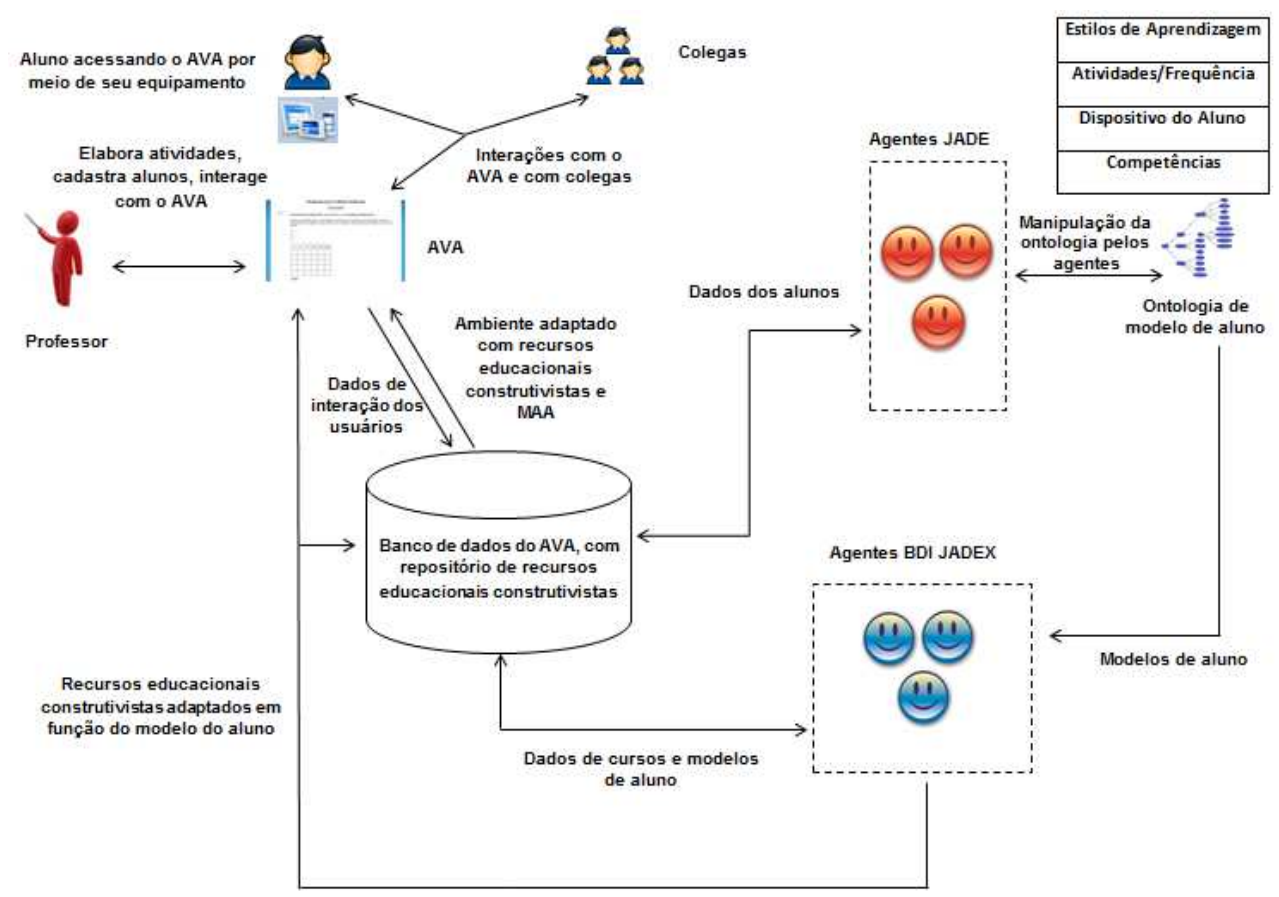

Figura 2. Arquitetura do AMPARA.

- Retrieved Profiles Agent: agente que faz a busca por dúvidas, erros dos alunos em atividades respondidas, avalia os níveis de habilidades e competências dos alunos, busca os estilos de aprendizagem dos mesmos, além das demais informações que compõem o MAA e por fim, mapeia esses dados em recursos de aprendizagem a serem disponibilizados no AVA, adaptando-o de acordo com o MAA. Estes recursos podem ser: colegas que possam tirar dúvidas, notificações de acompanhamento do aluno, sugestão de leituras e atividades baseadas em arquiteturas pedagógicas de acordo com o perfil de estudante. Com estas informações, este agente envia uma mensagem ao Recommended Resource Agent os dados dos alunos com os recursos associados a eles;

- Recommended Resources Agent: agente que obtém as informações do Retrieved Profiles Agent e executa o processo de mostrar no AVA os recursos para cada aluno em função de seu MAA.

\subsection{A Ontologia de Modelo Aberto de Aluno}

Outra importante etapa neste trabalho foi construir a ontologia de domínio que representa o modelo de aluno. Para o modelo de aluno foi utilizado o padrão IMS LIP, que é um padrão largamente usado hoje em dia e que permite extensões. Originalmente, o LIP tem 11 categorias (ou classes) de dados do estudante, mas apenas cinco foram usadas (Activity, Competency, Identification, Accessibility e Interest). Além disso, este padrão foi estendido para as necessidades deste trabalho, em três outras categorias: CCPP_Device, Learning_Styles e Frequency. A categoria CCPP_Device descreve os dispositivos utilizados pelos alunos. Assim como há padrões para o modelo do aluno, existem normas para a caracterização de dispositivos móveis, com as suas características como tamanho da tela, desempenho da CPU, memória, entre outros. Um dos mais usados atualmente e usado neste trabalho é o Composite Capabilities/Preference Profile (CC/PP) [CC/PP 2015]. Usando a categoria Frequency, esta descreve a frequência dos alunos no AVA e também é verificado se o aluno terminou ou não suas atividades. Finalmente, a categoria Learning_Styles contém informações sobre os estilos de aprendizagem de Felder-Silvernam e Honey-Alonso.

Na categoria Activity estão as informações acerca das atividades propostas pelo professor. As atividades, neste trabalho baseadas em Arquiteturas Pedagógicas, possuem metadados para documentá-las utilizando o padrão LTSC/IEEE Learning Object Metadata (LOM) [IEEE 2002]. Esses metadados são úteis para o mapeamento feito entre os campos do LOM nas Arquiteturas Pedagógicas e os estilos de aprendizagem do aluno, a fim de que as APs recomendadas aos alunos 
sejam mais precisas. Neste caso, as APs podem ser vistas como objetos de aprendizagem. Sendo assim, os campos do LOM que fazem parte das características das Arquiteturas Pedagógicas estão de acordo com um subconjunto dos campos de metadados selecionado a partir do trabalho de [Araújo et al. 2014], o qual propôs um mapeamento dos campos de metadados com relação às caraterísticas dos estilos de aprendizagem do aluno. A ontologia de modelo de aluno na classe Competency descreve o nível de habilidade de cada aluno, o que é útil para identificar os alunos e seus níveis de competências e habilidades em uma disciplina ou curso. Nesse trabalho, cada competência do aluno é composta por um conjunto de habilidades específicas. Os agentes fazem avaliações frequentes das atividades dos alunos, sendo uma avaliação formativa, obtendo seu desempenho e atualizando os níveis de habilidades dos alunos. Outras categorias utilizadas são Identification (para os dados pessoais do aluno), Accessibility (dados de acessibilidade do usuário, credenciais no AVA) e Interest (contendo os interesses dos estudantes).

A ontologia de modelo de aluno foi desenvolvida no editor Protégé [Protégé 2015] e tem três classes principais: Concept, cujas filhas são as subclasses AID (descrevendo os agentes que fazem parte do sistema multiagente) e Learner_LIP (contendo as categorias LIP utilizadas neste trabalho, além de CCPP_Device, Learning_Styles e Frequency); Predicate, responsável pelos fatos sobre o ambiente onde os agentes atuam; e AgentAction (contendo as ações que os agentes realizam no AVA). Todas estas três classes permitem que a ontologia seja integrada ao sistema multiagente. A Figura 3 mostra uma representação gráfica resumida da ontologia de MAA, onde aparece apenas a subclasse Learner_LIP de Concept, que descreve o padrão LIP estendido e apresenta os slots (propriedades ou atributos) das demais classes. Por exemplo, na classe Learning_Styles, uma propriedade é $f s \_l s \_a c t i v e \_r e f l e c t i v e$, indicando se o aluno tende a ser mais ativo ou reflexivo nos estilos de aprendizagem de Felder-Silvernam.

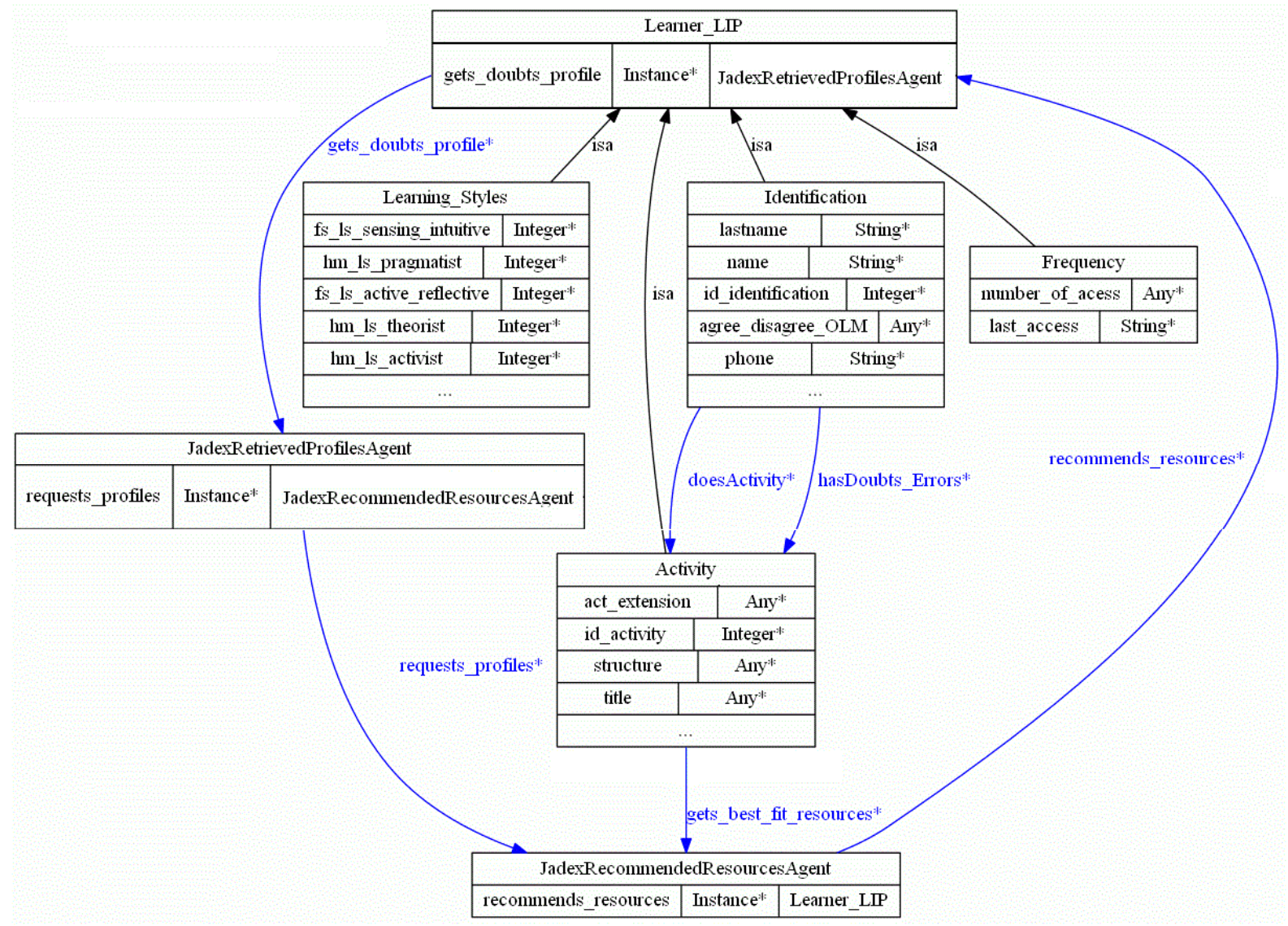

Figura 3. Representação gráfica resumida da Ontologia de MAA usada pelo AMPARA.

Os 2 agentes (subclasses de AID) apresentados na Figura 3 são o Retrieved Profiles Agent e Recommended Resources Agent, desenvolvidos no JADEX. As relações e regras entre as classes 
descrevem os facets da ontologia, representados por setas azuis e pretas (estas últimas indicando apenas a relação "isa", que significa "é um", de subclasse para superclasse). Como alguns exemplos de facets em setas azuis na Figura 3, o estudante faz uma atividade (doesActivity) e tem dúvidas ou erros (hasDoubts_Errors). Estes dois facets ligam as classes Identification e Activity. Além disso, o Recommended Resources Agent recomenda recursos (recommends_resources), por exemplo, APs de acodo com o perfil do estudante e as atividades que mais se ajustam a um determinado perfil de aluno (gets_best_fit_resources, que vem da classe Activity).

\section{Experimentos Realizados: MAA e Arquiteturas Pedagógicas Recomendadas}

O aluno pode acessar seu modelo por meio de um link no AVA Moodle ColabWeb (http://colabweb.ufam.edu.br/). É importante que o MAA seja facilmente compreendido pelo aluno. Assim, a Figura 4 mostra um exemplo de MAA apresentado aos alunos composto de três gráficos, que é o resultado das respostas dos alunos aos questionários. No primeiro gráfico são apresentados os estilos de aprendizagem de acordo com o questionário de Honey-Alonso, em porcentagens dos estilos Ativo, Reflexivo, Teórico e Pragmático. No segundo, os estilos de Felder-Silvernam (Ativo/Reflexivo, Sensorial/Intuitivo, Visual/Verbal e Sequencial/Global) são apresentados em valores ímpares que variam de -11 a +11 . Por exemplo, se um aluno tem no campo Visual/Verbal valor +7 , isso significa que ele é mais Visual. Se fosse -7 , ele seria mais Verbal. Finalmente, o terceiro gráfico mostra as habilidades do aluno em um curso específico, no nosso estudo de caso, Algoritmos e Programação.

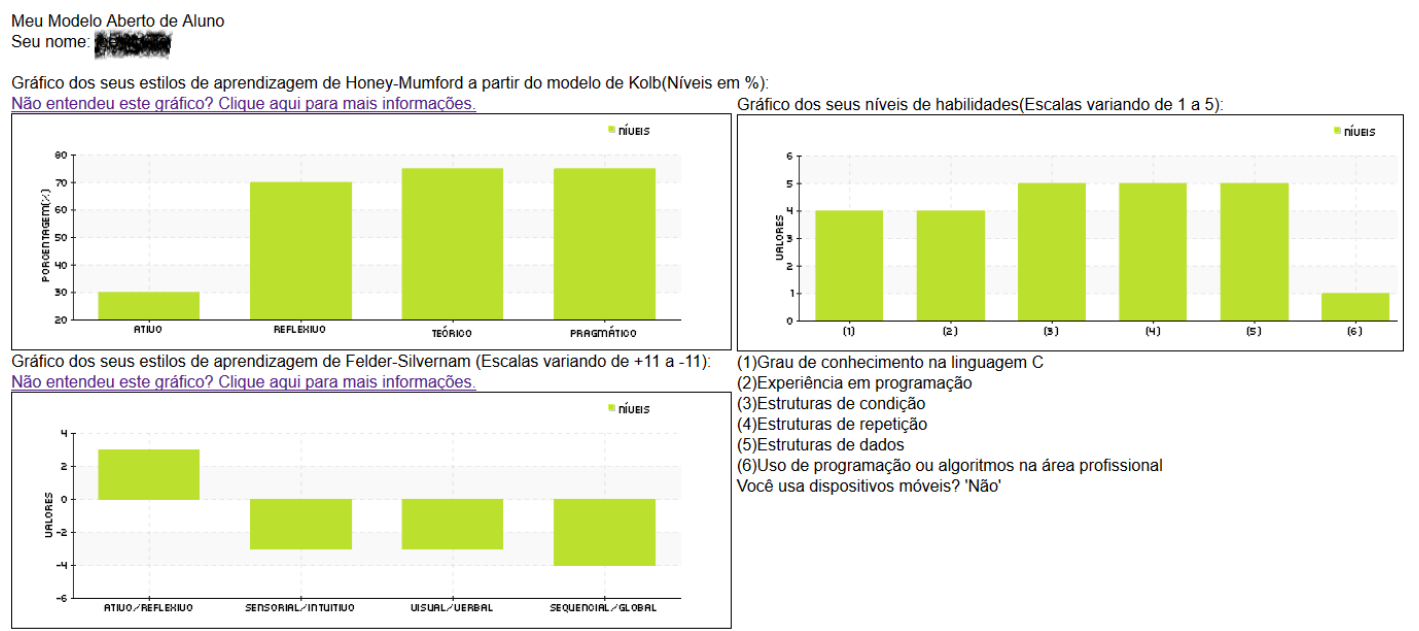

Figura 4. Apresentando o MAA aos estudantes.

No Moodle utilizado para testes, as adaptações de recursos (nesse caso as APs) foram em uma turma composta por 49 alunos da disciplina de Algoritmos e Programação do Instituto Federal do Amazonas (IFAM). Com a obtenção do perfil inicial de cada estudante a partir dos questionários de estilos de aprendizagem, foram recomendadas atividades de acordo com seus perfis. A Figura 5 mostra um exemplo de um debate de teses recomendado para alguns alunos, com a avaliação da resposta de um aluno por seu colega. Antes de fazerem as atividades, os alunos passaram por um treinamento, pois muitos não haviam experimentado no Moodle a atividade do tipo Laboratório de Avaliação (originalmente, chamada de Workshop). O Laboratório de Avaliação é uma atividade de avaliação entre pares (participantes) com uma vasta gama de opções, podendo ser utilizada como um debate de teses. Os participantes podem avaliar os trabalhos de outros participantes em diversos modos. O Laboratório de Avaliação também organiza o recebimento e a distribuição destas avaliações.

Na atividade de Laboratório de Avaliação do Moodle, a adaptação ocorre primeiramente utilizando a heurística de que pares de alunos para um debate de teses podem ser formados com alunos de perfis heterogêneos, conforme indicado em [Castro e Menezes, 2011]. Inicialmente, os perfis foram diferenciados por estilos de aprendizagem, mas as outras características dos alunos 
descritas em seus modelos também podem ser usadas. É importante ressaltar que estas recomendações de atividades são feitas pelo Recommended Resources Agent. Pode-se perceber nestes testes realizados que resultados satisfatórios estão sendo obtidos com a abordagem utilizada do AMPARA, uma vez que a recomendação de atividades baseadas em arquiteturas pedagógicas por meio de agentes e ontologia de MAA é uma técnica construtivista útil para melhorar o aprendizado do aluno e que incentiva a prática de atividades colaborativas em AVAs.

Os alunos participantes dos testes foram questionados sobre as atividades realizadas. Alguns dos depoimentos foram: "com a correção dos meus colegas aprendi mais"; "eu ajudei e fui ajudado com muitas correções"; "A avaliação das atividades dos colegas foi feita de forma bem coerente a mim testando meus conhecimentos e verificando o conhecimento dos meus colegas"; "primeira vez que presencio uma didática dessa forma, seria excelente se as demais matérias fossem dessa forma"; "Gostei, pois foi feito de maneira individual e ao mesmo tempo pudemos compartilhar conhecimento, e perceber que podemos chegar ao mesmo resultado usando métodos diferentes."; "eu achei interessante, principalmente porque vim zerado de algoritmos e linguagem $C$ e tive a capacidade de avaliar alguém que já conhecia, pelo nível que foi a resposta dele e acho que ele conhecia mais do que eu"; "nós que somos avaliados, é bom (...) estar na situação tipo professor podendo avaliar os colegas e poder interagir com as diferentes respostas que iremos encontrar"; "achei interessante para ver a dificuladde de cada aluno e assim, (...) poder ajudar (...) formando um único conhecimento geral". Uma crítica feita pelos alunos foi quanto à interface dos debates de teses apresentados no AVA.
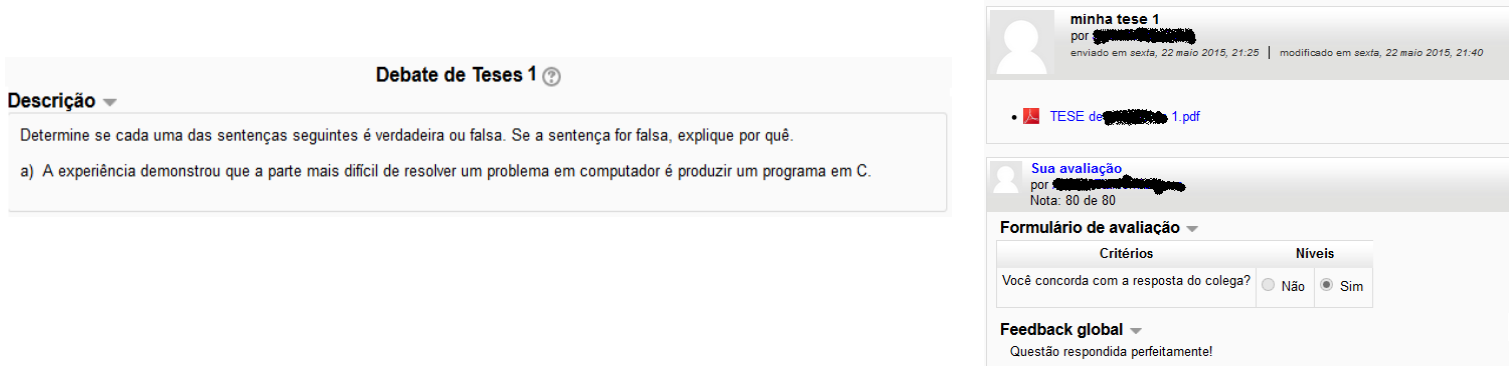

Figura 5. Exemplo da atividade debate de teses recomendada e sua avaliação por pares.

\section{Conclusões e Trabalhos Futuros}

Este artigo mostrou o arcabouço AMPARA, que é um modelo de adaptação de recursos em AVAs baseado na teoria construtivista da aprendizagem. Foram desenvolvidos um sistema multiagente e uma ontologia de Modelo Aberto de Aluno no padrão IMS LIP capazes de selecionar recursos educacionais adaptados no Moodle. Esta estratégia permite uma maior personalização dos recursos apresentados em função das características dos alunos. O processo de adaptação descrito na arquitetura do sistema aumenta a interação entre os alunos de um mesmo curso ou disciplina e a interação dos alunos com o AVA, aumentando assim o aprendizado efetivo e maiores acertos nas tarefas executadas. Os testes realizados mostraram que a recomendação de APs é uma solução que pode ajudar a construir o conhecimento do aluno de forma útil e eficaz. A abordagem de sistema multiagente, juntamente com o MAA, pode ser aplicada a outros AVAs, uma vez que os agentes e o ambiente educacional podem compartilhar a mesma base de dados. Como trabalhos futuros, a ideia é testar o sistema para adaptação de conteúdo em dispositivos móveis, visto que a ontologia já descreve os recursos do dispositivo móvel no padrão CC/PP e fazer ajustes na interface das atividades de debates de teses, tornando-as mais fáceis de usar.

\section{Referências}

Araújo, R. D. et al. (2014). "Autoria automática de objetos de aprendizagem a partir de captura multimídia e associação a estilos de aprendizagem”. Anais do XXV Simpósio Brasileiro de Informática na Educação (SBIE), Dourados, MS.

Alonso, C. e Gallego, D. (2015) Questionário Honey-Alonso de Estilos de Aprendizagem, http://www.estilosdeaprendizaje.es/chaea/chaeagrafp2.htm. 
Bastos Filho, O. C., Axt, M., Labidi, S. e Machado, C. T. (2006) "Sistema Inteligente de Desafios Abertos - IOCS: Uma proposta de adaptação dos padrões do Método Clínico Piagetiano em Plataforma Multiagentes". CINTED-UFRGS - Novas Tecnologias na Educação, V. 4 Nº 1, Julho.

Behar, P.; Bernardi, M; Silva, K. (2009). "Arquiteturas Pedagógicas para a Educação a Distância: a construção e validação de um objeto de aprendizagem”. RENOTE - Revista Novas Tecnologias na Educação, ISSN 1679-1916, CINTED-UFRGS, V.7. N.1.

Bremgartner, V.; Netto, J. M. e Menezes, C. S. (2014a). "Utilizando Agentes e Ontologia de Modelo de Aluno Aberto para Prover Adaptação de Conteúdos Construtivistas em Ambientes Virtuais de Aprendizagem". Anais do XXV Simpósio Brasileiro de Informática na Educação (SBIE), Dourados, MS.

Bremgartner, V.; Netto, J.M.; Menezes, C. S. (2014b) "Using Agents and Open Learner Model Ontology for Providing Constructive Adaptive Techniques in Virtual Learning Environments”. In: 14th IberoAmerican Conference on Artificial Intelligence - IBERAMIA 2014, Santiago.

Bull, S., Quigley, S. e Mabbott, A. (2006) "Computer-based Formative Assessment to Promote Reflection and Learner Autonomy”. Engineering Education, vol. 1, no. 1, pp. 8-18.

Carvalho, M. J. S; Nevado, R. A.; Menezes, C. S. (2005). “Arquiteturas Pedagógicas para Educação a Distância: Concepções e Suporte Telemático”. Anais - XVI Simpósio Brasileiro de Informática na Educação, 1, 362-372.

Castro, A. N. e Menezes, C. S. (2011) “Aprendizagem Colaborativa com Suporte Computacional”. In: Pimentel, M. e Fuks, H. Sistemas Colaborativos. Rio de Janeiro: Campus. ISBN: 978-85-352-4669-8.

CC/PP. (2015) Composite Capabilities/Preference Profiles, www.w3.org/Mobile/CCPP/

Corte Real, L. M. e Corbellini, S. (2011). "Proposta de uso de WIKI como Arquitetura Pedagógica: cooperação". Anais do XVII Workshop de Informática na Escola (WIE), Aracaju, SE.

Felder, R. M. e Silverman, L. K. (1988). "Learning and teaching styles in engineering education". Engineering Education, 78, pp. 674-681.

Ferrari, M; Oliveira, O. L e Menezes, C. S. (2014). “AProAp - Um ambiente para projetos de aprendizagem”. Anais do XXV Simpósio Brasileiro de Informática na Educação (SBIE), Dourados, MS.

Georgeff, M., Pell, B., Pollack, M., Tambe, M. e Wooldridge, M. (1999) “The Belief-Desire-Intention Model of Agency". In: Intelligent Agents V, LNAI 1555, Springer, pp. 1-10.

IEEE, LTSC. (2002). Draft Standard for Learning Technology - Learning Object Metadata. Technical report, IEEE Standards Department, New York.

JADE. (2015) Java Agent DEvelopment Framework, www.jade.tilab.com/

JADEX. (2015) JADEX Active Components, www.activecomponents.org/bin/view/About/Features

LIP. (2015) Learner Information Package Specification 1.0.1, www.imsglobal.org/profiles/lipinfo01.html\#5.1/

Marcon, K.; Machado, J. B.; Carvalho, M. J. S. (2012). “Arquiteturas pedagógicas e redes sociais: uma experiência no Facebook”. In: Anais do XVIII Congresso Brasileiro de Informática na Educação: Rio de Janeiro, RJ.

Moodle (2015) A Free, Open Source Course Management System for Online Learning, www.moodle.org/

Oliveira, E. A. e Tedesco, P. (2009) "i-Collaboration in Practice: Results from our Investigation within the Cleverpal Environment". Proceedings of IEEE International Conference on Intelligent Computing and Intelligent Systems (ICIS), pp. 347-351.

Panceri, S. S. e Menezes, C. S. (2014). “ALPES: Um sistema multiagente para análise de produções textuais no contexto de um Debate de Teses". Anais do XXV Simpósio Brasileiro de Informática na Educação (SBIE), Dourados, MS.

Protégé. (2015) The Ontology Editor, www.protege.stanford.edu/

Saccol, A. Z., Kich, M., Schlemmer, E., Reinhard, N., Barbosa, J. L. V. e Hahn, R. (2009) “A Framework for the Design of Ubiquitous Learning Applications”. In 42nd Hawaii International Conference on System Sciences Proceedings.

Viccari, R. M. e Giraffa, L. M. M. (2003) "Fundamentos dos Sistemas Tutores Inteligentes”. In: Barone, D. (Org). Sociedades artificiais: a nova fronteira da inteligência das máquinas. Porto Alegre: Bookman. ISBN: 85-363-0124-4. 